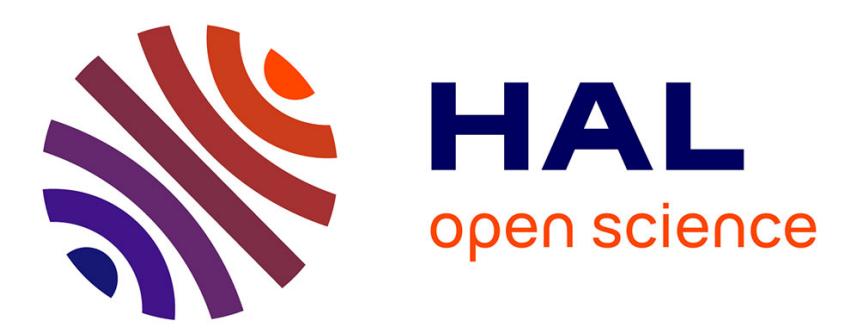

\title{
Effet de la température des racines sur leur respiration et sur la croissance des plantules de deux hybrides de maïs
}

\author{
Jean-Sylvain Frossard, Maurice M. Crocombette
}

\section{- To cite this version:}

Jean-Sylvain Frossard, Maurice M. Crocombette. Effet de la température des racines sur leur respiration et sur la croissance des plantules de deux hybrides de maïs. Agronomie, 1985, 5 (8), pp.719-725. hal-00884804

\section{HAL Id: hal-00884804 https://hal.science/hal-00884804}

Submitted on 1 Jan 1985

HAL is a multi-disciplinary open access archive for the deposit and dissemination of scientific research documents, whether they are published or not. The documents may come from teaching and research institutions in France or abroad, or from public or private research centers.
L'archive ouverte pluridisciplinaire HAL, est destinée au dépôt et à la diffusion de documents scientifiques de niveau recherche, publiés ou non, émanant des établissements d'enseignement et de recherche français ou étrangers, des laboratoires publics ou privés. 


\title{
Effet de la température des racines sur leur res- piration et sur la croissance des plantules de deux hybrides de maïs
}

\author{
Jean-Sylvain FROSSARD \\ avec la collaboration technique de Maurice CROCOMBETTE \\ I.N.R.A., Laboratoire de Bioclimatologie, Domaine de Crouelle, F-63039 Clermont-Ferrand
}

RÉSUMÉ

\begin{abstract}
L'effet de la température racinaire sur la respiration des racines et la croissance de la plante a été étudié, à court terme, sur de jeunes plants de maïs âgés de $14 \mathrm{j}$, pour un hybride de type denté et un hybride de type corné. Les résuitats montrent que la modification de la température a un effet immédiat sur la respiration pour les deux hybrides. On ne constate de différences entre hybrides qu'au-delà d'un certain temps. D'une façon générale, la croissance de l'hybride de type denté est plus affectée par la température $\left(15,10^{\circ} \mathrm{C}\right)$ que celle de l'hybride de type corné. Seuls les paramètres de croissance décrivant les racines permettent de différencier les deux génotypes.
\end{abstract}

Mots clés additionnels : Teneur en eau, tolérance au froid, ajustement, adaptation.

Root temperature effect on root respiration and growth in maize seedlings of two grain types.

\begin{abstract}
The effect of root temperature on root respiration and growth was studied on 14-day old maize plants of two grain types (flint and dent). The plants were grown on nutrient solution in a growth cabinet at constant temperature $\left(20^{\circ} \mathrm{C}\right.$ ). At 14 days the plants were transfered to different root temperatures : 10, 15, 20 (control) and $25^{\circ} \mathrm{C}$. The study was carried out over $48 \mathrm{~h}$. The mean level and the diurnal variation of root respiration increased with temperature. The variation of root respiration with temperature over the day and night was not uniform in the two types. Two-way variance analysis revealed that, for root respiration, i.e. production of elemental energy for other functions of the root system, the differences between genotypes were more in quick adaptation effect than in stress effect. For growth, the main differences were for the root system, dry matter accumulation and water content. In this case, the differences between genotypes were mainly in stress effect.
\end{abstract}

Additional key words : Water content, temperature stress, temperature adaptation.

\section{INTRODUCTION}

Un des obstacles au développement de la culture du maïs dans la partie septentrionale de la France, est lié à la fréquence de températures basses au printemps lors de l'installation de la culture. Les généticiens ont exploité les différences de sensibilité qui existent entre divers hybrides suivant leur origine (CAUDERON, 1958).

Aux stades jeunes, de nombreux travaux ont souligné l'influence plus grande de la température du milieu souterrain sur la croissance et le développement (Allmaras et al., 1964 ; DUBURCQ et al., 1983) ou l'activité métabolique des différentes parties de la plante (GrobBelaAR, 1963 ; CHAillou et al., 1976 ; McADAM \& HAYES, 1981).
L'abaissement de la température au niveau racinaire modifie aussi plus ou moins rapidement le bilan hydrique des feuilles (KLEINENDORST \& BROUWER, 1970), la structuration du système racinaire (BEAUCHAMP \& LATHWELL, 1966), la teneur en sucres solubles des pointes de racines (CRAWFORD \& HUXTER, 1977), la teneur en eau de la plante (CHAILlou et al., 1976).

Bien que ces effets soient systématiques quel que soit le génotype, on observe une atténuation des effets pour les génotypes plus "tolérants" au froid (STAMP, 1978; AKIMOVA \& RODCHENKO, 1977 ; BURBANOVA \& RODCHENKO, 1980). Cette plus grande tolérance aux basses températures est plus le fait des génotypes de type corné que des génotypes de type denté (STAMP, 1978; BLONDON et al., 1981; DERIEUX \& BONHOMME, 1982). 
Toute modification de l'environnement physique de la plante entraîne deux types d'effets sur son fonctionnement. A très court terme, la modification du fonctionnement de la plante est liée à son "équipement " (enzymatique, par exemple) présent au moment de l'application de la contrainte. A plus long terme, la modification du fonctionnement de la plante provient d'une modification de son « équipement", modification induite par la contrainte. En reprenant les définitions proposées par BoURdU \& PRIOUL (1974) lors d'une étude sur la photosynthèse, nous emploierons le terme d' " effet ajustement " pour le $1^{\text {er }}$ type et celui $d^{\prime}$ ' effet adaptation » pour le $2^{e}$ type.

Toutes les études que nous avons rapportées cidessus ont été réalisées dans un intervalle de temps plus ou moins long, de plusieurs jours à quelques semaines (sauf pour l'étude de CRAWFORD \& HUXTER, 1977). Les résultats obtenus offrent donc très rarement la possibilité de différencier un « effet ajustement » d'un « effet adaptation ».

L'étude présentée ici a pour but de préciser les différences de comportement à la température au niveau des racines, à court terme, donc de privilégier, $a$ priori, l'« effet ajustement ", de 2 génotypes aux exigences thermiques très différentes.

Nous analyserons les effets thermiques à travers l'étude de l'évolution :

- de l'activité racinaire instantanée, appréciée par la respiration ;

- de paramètres de croissance.

En outre, ne disposant d'aucune information précise sur l'effet à court terme de la température racinaire sur la photosynthèse et la transpiration, nous présenterons quelques résultats concernant ces 2 phénomènes, avec pour seul objectif d'affiner l'étude proposée ci-dessus.

\section{MATÉRIEL ET MÉTHODES}

\section{A. Matériel végétal}

Il est constitué par 2 hybrides simples :

- F7 $\times$ F2, de type corné, tolérant aux températures basses au printemps en conditions normales de culture, au champ ;

- WH $\times$ WJ, de type denté, sensible au froid printanier.

Après semis et germination sur sable humide $(8 \mathrm{j}$ à $20^{\circ} \mathrm{C}$, avec $12 \mathrm{~h}$ de lumière), les plantes sont élevées pendant $14 \mathrm{j}$ sur solution nutritive aérée, en chambre climatisée, avec les conditions suivantes :

- durée de la photophase et de la scotophase : $12 \mathrm{~h}$;

- rayonnement pendant la photophase: 130 W.m . $^{-2}$ (PAR) ;

- température de l'air et de la solution :

$20 \pm 0,5^{\circ} \mathrm{C}$;

— humidité relative : $70 \pm 5$ p. 100.

La température et l'humidité relative sont constantes jour et nuit.

La composition de la solution nutritive est similaire à celle déjà utilisée pour d'autres espèces (FROSSARD, 1976) avec un $\mathrm{pH}$ ajusté à 5,0 .
$\mathrm{Au} 14^{\mathrm{e}} \mathrm{j}$, les plantes sont transférées sur des bacs de solution nutritive (composition identique à celle des conditions d'élevage) dont la température est réglée aux valeurs suivantes : $10,15,20$ (témoin) et $25^{\circ} \mathrm{C}$ (précision de la régulation : $\pm 0,1^{\circ} \mathrm{C}$ ).

$\mathrm{Au}$ moment des expériences, les plantes ont, en moyenne, 3 feuilles complètement déroulées.

\section{B. Mesure de la respiration racinaire}

La respiration est appréciée par l'absorption d'oxygène mesurée au moyen d'une électrode polarographique ; les mesures sont réalisées en continu avec un respiromètre automatique décrit par ailleurs (FROSSARD, 1976).

Les plantes sont transférées dans le respiromètre, à l'âge de $14 \mathrm{j}$, à la température de mesure $(10,15,20$ et $25^{\circ} \mathrm{C}$ pour les racines), sans période d'adaptation préalable. Les autres conditions climatiques sont identiques à celles de la période d'élevage. Le nombre d'individus par traitement (température $\times$ génotype) est de 4 . La respiration racinaire est mesurée pour chaque individu, de façon indépendante.

\section{Analyse de la croissance}

$48 \mathrm{~h}$ après transfert sur les bacs de solution thermostatée, on mesure de façon destructrice différents paramètres dont la liste est donnée au tableau 1 . Il y a 8 répétitions par traitement.

TABLEAU 1

Signification des symboles utilisés dans les tableaux. Meaning of symbols.

\begin{tabular}{|c|c|c|}
\hline Symbole & Signification & Unités \\
\hline PFT & $\begin{array}{l}\text { poids de matière fraîche de la plante } \\
\text { plant fresh weight }\end{array}$ & g \\
\hline PFR & $\begin{array}{l}\text { poids de matière fraîche des racines } \\
\text { fresh weight of the roots }\end{array}$ & $\mathrm{g}$ \\
\hline PFPA & $\begin{array}{l}\text { poids de matière fraîche des parties aériennes } \\
\text { fresh weight of the shoot }\end{array}$ & $\mathrm{g}$ \\
\hline SF & $\begin{array}{l}\text { surface foliaire } \\
\text { leaf area }\end{array}$ & $\mathrm{cm}^{2}$ \\
\hline PST & $\begin{array}{l}\text { poids de matière sèche de la plante } \\
\text { dry matter weight of the plant }\end{array}$ & g \\
\hline PSR & $\begin{array}{l}\text { poids de matière sèche des racines } \\
\text { dry matter weight of the roots }\end{array}$ & $\mathrm{g}$ \\
\hline PSPA & $\begin{array}{l}\text { poids de matière sèche des parties aériennes } \\
\text { dry matter weight of the shoots }\end{array}$ & g \\
\hline TET & $\begin{array}{l}\text { teneur en eau de la plante } \\
\text { water content of the whole plant } \\
\text { TET }=\frac{\text { PFT }- \text { PST }}{\text { PST }}\end{array}$ & $\%$ \\
\hline TER & $\begin{array}{l}\text { teneur en eau des racines } \\
\text { water content of the roots } \\
\mathrm{TER}=\frac{\mathrm{PFR}-\mathrm{PSR}}{\mathrm{PSR}}\end{array}$ & $\%$ \\
\hline TEPA & $\begin{array}{l}\text { teneur en eau des parties aériennes } \\
\text { water content of the shoots } \\
\text { TEPA }=\frac{\text { PFPA - PSPA }}{\text { PSPA }}\end{array}$ & $\%$ \\
\hline
\end{tabular}




\section{Mesure de la photosynthèse et de la transpiration}

La photosynthèse et la transpiration sont mesurées dans une chambre d'assimilation et de transpiration ou CAT (CHATENOUD, 1982). La photosynthèse est mesurée au moyen d'un analyseur différentiel à $\mathrm{CO}_{2}$ à rayonnement infrarouge. La transpiration est appréciée par la mesure différentielle de l'hygrométrie de l'air au moyen de 2 sondes capacitives.

Les plantes sont transférées dans la CAT au $14^{\mathrm{e}} \mathrm{j}$, les racines étant toujours à $20^{\circ} \mathrm{C}$. Toutes les autres conditions sont identiques à celles de la mesure de la respiration racinaire ou de l'analyse de croissance.

On mesure la photosynthèse et la transpiration dans ces conditions pendant une photophase. Deux heures avant le début de la $2^{\mathrm{e}}$ photophase, la température de la solution nutritive est ajustée à une autre température (régulation thermique à $0,1^{\circ} \mathrm{C}$ ); on mesure la photosynthèse et la transpiration dans ces nouvelles conditions, après avoir mesuré la surface foliaire de façon non destructive.

Recherchant une information plus qualitative que quantitative (la température du milieu racinaire modifie-t-elle la photosynthèse et la transpiration?), nous avons limité le nombre de traitements et le nombre de répétitions par traitement : 2 températures, 20 et $10^{\circ} \mathrm{C}, 3$ répétitions par traitement (température $x$ génotype).

\section{RÉSULTATS ET DISCUSSION}

\section{A. Evolution de la respiration racinaire suivant la température}

Cette évolution est représentée sur la figure 1. On observe que, quel que soit le génotype, le niveau moyen et l'amplitude nycthémérale augmentent avec la température. Cependant, quelle que soit la température, l'intensité respiratoire (IR) de WH $\times$ WJ est toujours inférieure à celle de F7 $\times$ F2.
A partir de la variation de l'amplitude nycthémérale observée et d'autres résultats obtenus par ailleurs, on peut formuler quelques hypothèses sur l'état des relations trophiques entre partie aérienne et partie souterraine.

A $20^{\circ} \mathrm{C}$ et à $25^{\circ} \mathrm{C}$, on peut avancer que le métabolisme énergétique des racines est sous la dépendance directe de la fourniture d'assimilats par l'appareil foliaire, comme cela a déjà été signalé (FrOSSARD, 1981).

Par analogie, on pourrait penser qu'à des températures plus basses l'approvisionnement en assimilats limite la respiration racinaire. Or, comme l'avait relevé GROBBELAAR (1963), à long terme, on peut remarquer que la température de $10^{\circ} \mathrm{C}$ appliquée aux racines ne modifie pas la photosynthèse (fig. 2). En l'absence d'autres éléments, on peut donc attribuer la décroissance de l'amplitude nycthémérale et du niveau général de la respiration à une limitation dans l'utilisation des assimilats reçus, limitation liée à un ralentissement des vitesses de réactions des systèmes enzymatiques (SUTCLIFFE, 1977).

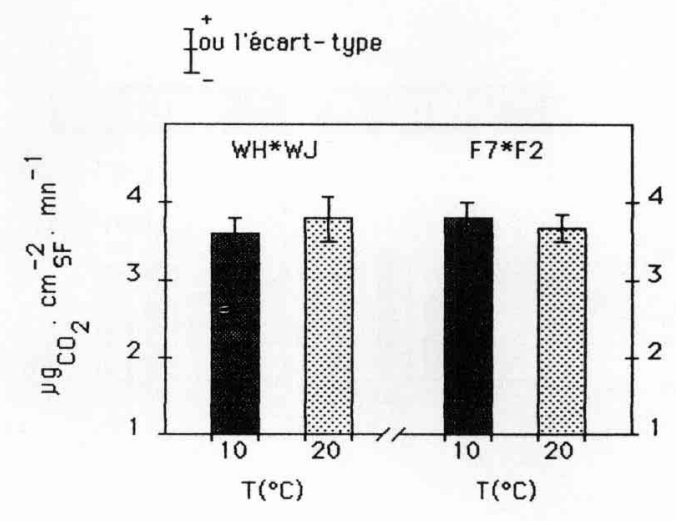

Figure 2

Photosynthèse à deux températures racinaires pour les deux hybrides.

Photosynthesis at two different root temperatures.

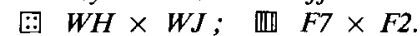

Figure 1

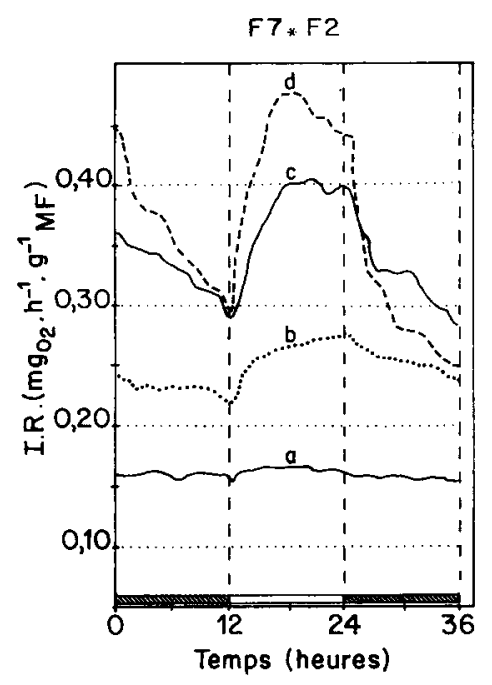

Evolution de la respiration racinaire au cours du temps suivant la température du milieu : (a) $10^{\circ} \mathrm{C}$, (b) $15^{\circ} \mathrm{C}$, (c) $20^{\circ} \mathrm{C}$, (d) $25^{\circ} \mathrm{C}$.
Root respiration versus time at different root temperatures : (a) $10{ }^{\circ} \mathrm{C}$, (b) $15{ }^{\circ} \mathrm{C}$, (c) $20{ }^{\circ} \mathrm{C}$, (d) $25{ }^{\circ} \mathrm{C}$. 
L'évolution du niveau moyen de la respiration en fonction de la température suggère certains commentaires.

En conditions aérobies, la respiration est la principale source d'ATP, « énergie primaire», pour un organe non chlorophyllien. Toute diminution pourrait être à l'origine de la décroissance de fonctions fortement consommatrices de cette énergie primaire observée par ailleurs telles l'absorption minérale ou la synthèse de protéines (GROBBELAAR, 1963 ; CHAILLOU et al., 1976 ; CAREY \& BERRY, 1978 ; BURBANOVA \& RODCHENKO, 1980 ; BRAVO \& URIBE, 1981). De plus, les valeurs plus basses observées pour $\mathrm{WH} \times \mathrm{WJ}$ sont en bon accord avec l'activité enzymatique systématiquement plus faible des génotypes sensibles aux basses températures (AKIMOVA \& RODCHENKO, 1977 ; BURBANOVA \& RODCHENKO, 1980).

L'étude des quantités d'oxygène absorbé suivant les phases d'obscurité et de lumière dont les variations sont représentées sur la figure 3 révèle, au-delà des différences variétales, qu'il n'existe pas une loi d'action unique de la température sur la respiration racinaire : il y a lieu de distinguer les périodes lumineuses des périodes obscures.
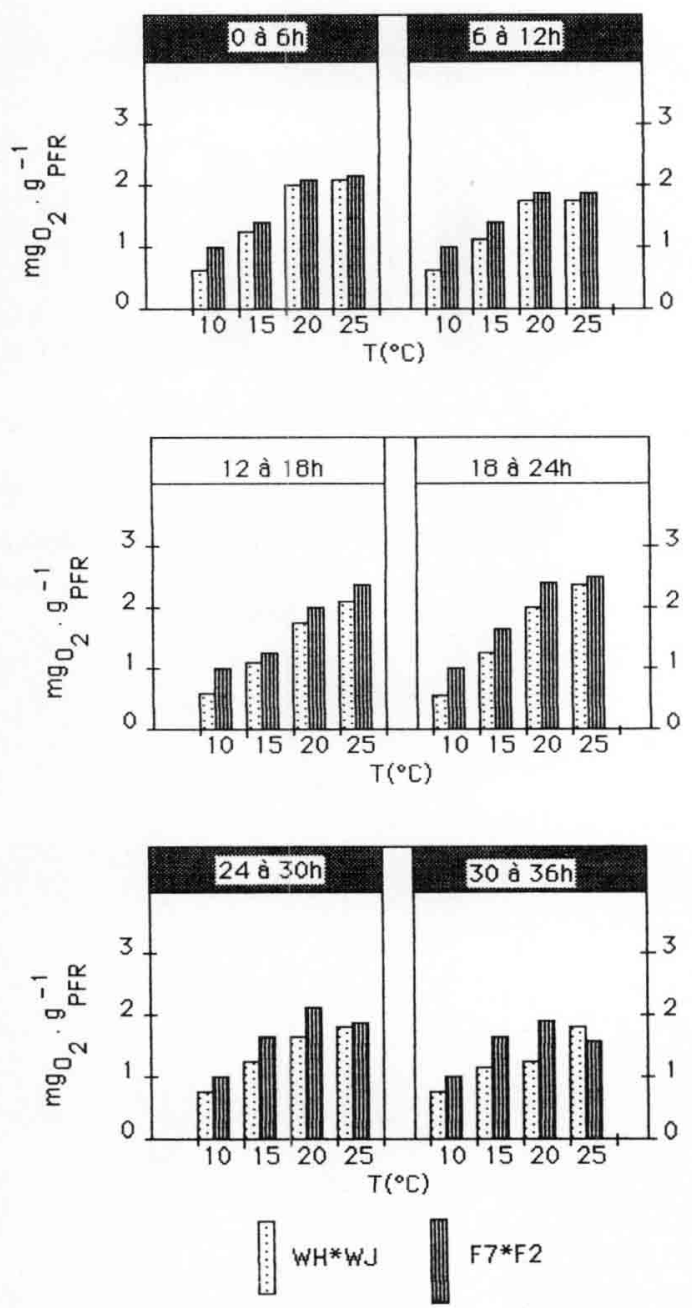

Figure 3

Variations de la quantité d'oxygène absorbé au cours des scotophases $(0$ à $12 \mathrm{~h}$ et 24 à $36 \mathrm{~h})$ et de la photophase $(12$ à $24 \mathrm{~h})$ pour $W H \times W J$ et $F 7 \times F 2$.

Amount of oxvgen ahsorbed during night (from 0 to $12 \mathrm{~h}$ and from 24 to $36 \mathrm{~h}$ ) and day (from 12 to $24 \mathrm{~h}$ ) for $\mathrm{WH} \times \mathrm{WJ}$ and $\mathrm{F7} \times \mathrm{F2}$.
Dans notre cas, on ne peut pas définir un $\mathrm{Q}_{10}$ unique et encore moins avancer que le $\mathrm{Q}_{10}$ de la respiration est égal à 2 dans la gamme de températures non létales comme on l'avance généralement (SUTCLIFFE, 1977). Ce fait a déjà été souligné pour la respiration nocturne du trèfle blanc (FUKAI \& SILSBURY, 1977). Comme l'avaient montré leurs résultats, on peut penser que la respiration d'un organe ou d'un organisme, étant la résultante d'un certain nombre d'interactions (disponibilité en substrat respiratoire, demande énergétique pour des besoins de croissance ou d'entretien...), elle ne réagit pas à la température de façon simple et similaire à celle des mitochondries ou de micro-organismes sur lesquels ont été généralement étudiées les lois d'action de la température et a été défini le $\mathrm{Q}_{10}$.

Les résultats d'une analyse de variance à 2 critères présentés au tableau 2 permettent de constater l'instauration progressive au cours du temps d'une discrimination significative suivant le génotype alors que l'effet de la température est immédiatement significatif.

Ces éléments permettent d'avancer que les différences entre les 2 génotypes, pour le catabolisme énergétique primaire, se situeraient plus dans un effet " adaptation » rapide que dans un effet « ajustement ».

\section{B. Effet de la température sur la croissance}

Les résultats des mesures des différents paramètres de croissance sont présentés au tableau 3. On remarque que plus la température est élevée plus les poids de matière fraîche (PFT, PFR et PFPA) sont élevés, alors que c'est exactement l'inverse pour les poids de matière sèche (PST, PSR et PSPA).

De façon générale, la diminution de la teneur en eau avec les températures permet d'expliquer ces évolutions contraires. Cette observation est similaire à celle relevée par CHAILLOU et al. (1976) dans le cas où la température des parties aériennes est nettement différente de celle appliquée aux racines $\left(22^{\circ} \mathrm{C}\right.$ et $12{ }^{\circ} \mathrm{C}$ ).

L'évolution de quelques paramètres de croissance est représentée sur la figure 4 . On relève dans tous les cas un effet beaucoup plus net des températures pour $\mathrm{WH} \times \mathrm{WJ}$ que pour F7 $\times$ F2. La discrimination entre les 2 hybrides est très forte pour le rapport $\mathrm{PST} / \mathrm{SF}$.

\section{TABLEAU 2}

Résultats d'analyses de variance à 2 critères portant sur la quantité d'oxygène absorbé $\left({ }^{* *}\right.$ : l'effet est significatif au niveau $\left.1 \%\right)$.

Two-way variance analysis of oxygen absorbed during the scotophase or the photophase $1^{* *}:$ significant effect at $1 \%$ level).

\begin{tabular}{|c|c|c|c|c|c|c|}
\hline Type d'effet & & & & & & \\
\hline Interaction & NS & NS & NS & $* *$ & NS & $* *$ \\
\hline Génotype & NS & NS & $* *$ & $* *$ & $* *$ & $* *$ \\
\hline Température & $* *$ & $* *$ & $* *$ & $* *$ & $* *$ & $* *$ \\
\hline Temps en $\mathrm{h}$ & & & & & & \\
\hline
\end{tabular}


TABLEAU 3

Valeurs moyennes des paramètres de croissance pour $\mathrm{WH} \times \mathrm{WJ}$ et pour $\mathrm{F7} \times \mathrm{F2}$ Means of growth parameters for $W H \times W J$ and for $F 7 \times F 2$.

\begin{tabular}{|c|c|c|c|c|c|c|c|c|c|c|}
\hline Hybrides & & & $\mathrm{WH} \times \mathrm{WJ}$ & & & & & $\mathrm{F} 7 \times \mathrm{F} 2$ & & \\
\hline Age en jours & 14 & 16 & 16 & 16 & 16 & 14 & 16 & 16 & 16 & 16 \\
\hline Température & 20 & 10 & 15 & 20 & 25 & 20 & 10 & 15 & 20 & 25 \\
\hline PFT & 3,44 & 4,07 & 5,98 & 6,02 & 7,84 & 3,75 & 5,74 & 4,69 & 6,53 & 5,60 \\
\hline PFR & 1,92 & 1,92 & 3,26 & 3,06 & 4,38 & 1,84 & 2,39 & 2,07 & 3,07 & 2,72 \\
\hline PFPA & 1,52 & 2,15 & 2,72 & 2,87 & 3,45 & 1,91 & 3,35 & 2,62 & 3,46 & 2,88 \\
\hline SF & 40,1 & 51,8 & 68,5 & 68,6 & 76,7 & 48,6 & 76,6 & 63,6 & 79,6 & 68,7 \\
\hline PST & 0,29 & 0,61 & 0,48 & 0,41 & 0,43 & 0,30 & 0,47 & 0,37 & 0,52 & 0,33 \\
\hline PSR & 0,14 & 0,27 & 0,22 & 0,17 & 0,18 & 0,11 & 0,15 & 0,13 & 0,18 & 0,10 \\
\hline PSPA & 0,16 & 0,34 & 0,26 & 0,24 & 0,25 & 0,19 & 0,32 & 0,24 & 0,34 & 0,22 \\
\hline PSR/PSPA & 0,87 & 0,80 & 0,84 & 0,70 & 0,73 & 0,55 & 0,48 & 0,55 & 0,56 & 0,46 \\
\hline $\mathrm{PSR} / \mathrm{PST}$ & 0,46 & 0,45 & 0,45 & 0,41 & 0,42 & 0,35 & 0,33 & 0,35 & 0,36 & 0,31 \\
\hline$(\mathrm{PST} / \mathrm{SF}) 1000$ & 7,2 & 11,7 & 7,1 & 5,9 & 5,6 & 6,1 & 6,1 & 6,1 & 6,1 & 5,1 \\
\hline TET & 1094 & 556 & 1146 & 1375 & 1727 & 1111 & 1007 & 1179 & 1142 & 1626 \\
\hline TER & 1359 & 557 & 1404 & 1796 & 2334 & 1591 & 1410 & 1483 & 1573 & 2619 \\
\hline TEPA & 880 & 519 & 939 & 1085 & 1286 & 911 & 842 & 1011 & 909 & 1191 \\
\hline
\end{tabular}

Ayant constaté que la température du milieu racinaire avait peu d'effet sur la croissance en surface des feuilles, d'une part (fig. 4), et sur la photosynthèse par unité de surface, d'autre part (cf. $\S$ A, fig. 2), on peut avancer que ce qui différencie $\mathrm{WH} \times \mathrm{WJ}$ de F7 $\times$ F2 à basse température est l'accumulation de matière sèche. A partir de résultats obtenus pour la mesure de la respiration racinaire (cf. $\S$ A. fig. 3), on peut remarquer que la plus grande accumulation de matière sèche relevée pour $\mathrm{WH} \times \mathrm{WJ}$ coïncide avec le niveau de respiration le moins élevé.

Les résultats concernant la teneur en eau des racines, des parties aériennes et de la plante entière sont représentés sur la figure 5 . Comme pour les autres paramètres, cette évolution est beaucoup plus sensible à la température pour $\mathrm{WH} \times \mathrm{WJ}$ que pour $\mathrm{F} 7 \times \mathrm{F} 2$. Pour ce génotype, seule la température de $25^{\circ} \mathrm{C}$ modifie la teneur en eau ; cet effet est principalement visible pour les racines.

La transpiration n'est pas affectée par la température racinaire dans l'intervalle de temps des mesures (fig. 6). L'effet observé sur les teneurs en eau pourrait provenir de la limitation de l'absorption hydrique par

Figure 4

Poids de matière sèche des racines (PSR), poids total de matière sèche (PST) et rapport poids total de matière sèche-surface foliaire $(P S T / S F)$ suivant la température, exprimés en p. 100 du témoin $\left(20^{\circ} \mathrm{C}\right)$.

PSR, PST and PST/SF at different root temperatures, per cent of control $\left(20^{\circ} \mathrm{C}\right)$.
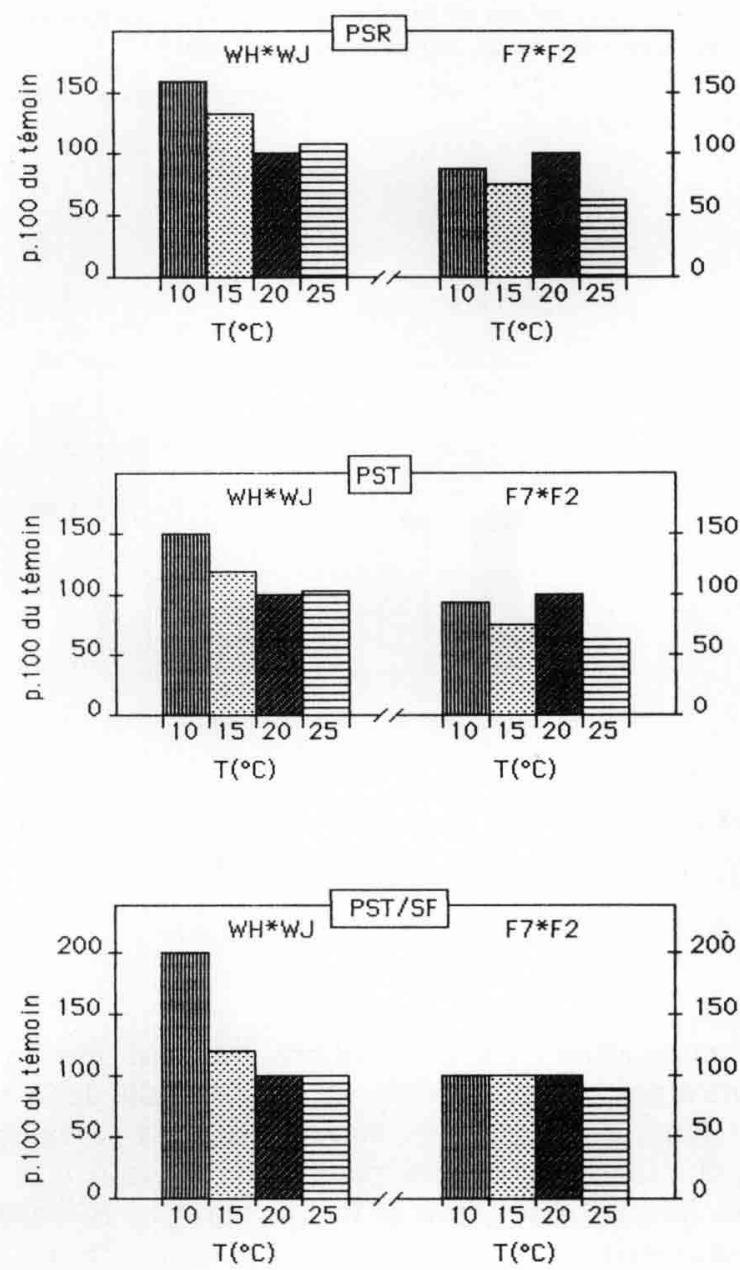

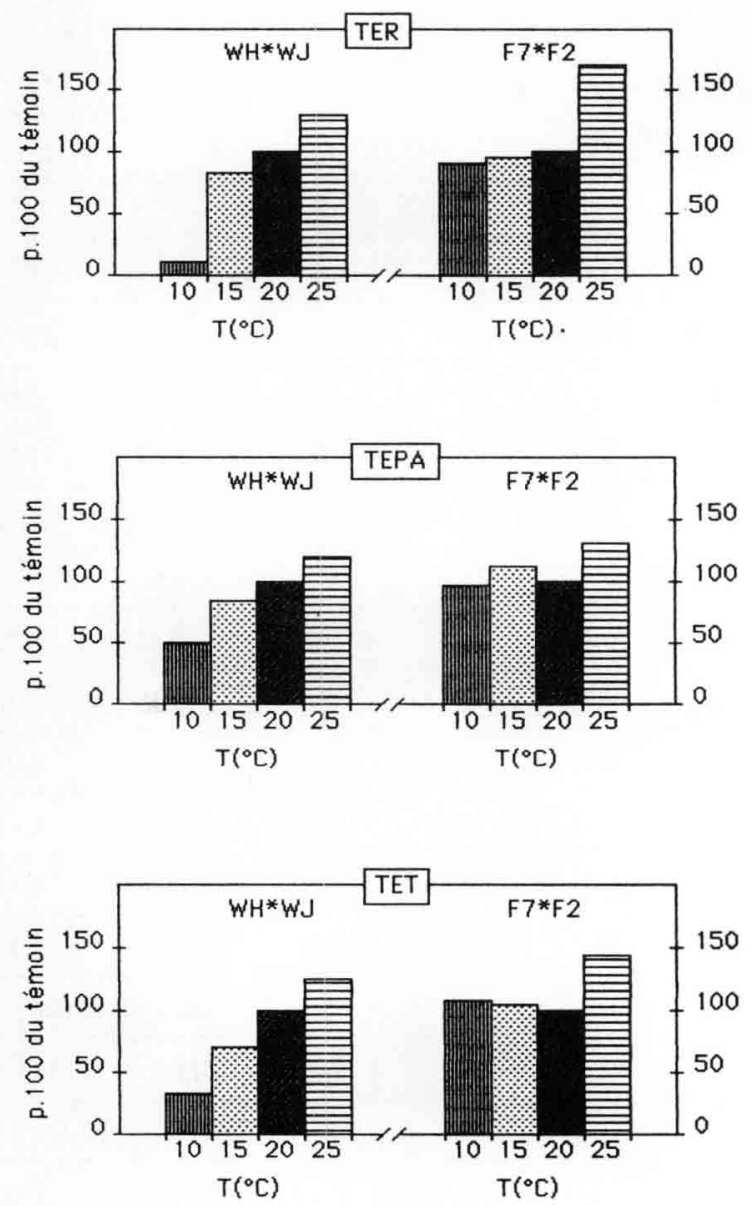

Figure 5

Teneur en eau des racines (TER), teneur en eau de la partie aérienne (TEPA) et teneur en eau de la plante entière (TET) suivant la température, exprimées en p. 100 du témoin $\left(20^{\circ} \mathrm{C}\right)$.

$T E R, T E P A$ and TET at different root temperatures, per cent of control $\left(20 .{ }^{\circ} \mathrm{C}\right)$.

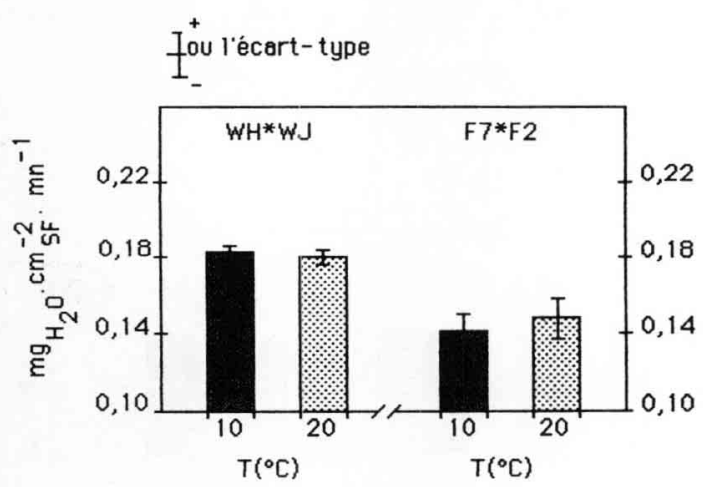

Figure 6

Transpiration à deux températures racinaires pour les deux hybrides. Transpiration at two different root temperatures.

la température. Ce déséquilibre ne peut être que momentané puisqu'à terme, sur un intervalle de temps plus long, il y a équilibre entre absorption et transpiration : une température peu élevée du milieu racinaire limite l'absorption et la transpiration (GROBBELAAR, 1963).
L'analyse de variance à 2 critères, dont les résultats sont présentés au tableau 4 , révèle que la température des racines a un effet hautement significatif sur tous les paramètres de croissance hormis la surface foliaire. L'“ effet génotype » n'est significatif que lorsqu'il s'applique à des paramètres décrivant les racines.

Les différences de croissance générale constatées par STAMP (1978) ou BLONDON et al. (1980) entre des génotypes de type corné et des génotypes de type denté ne se retrouvent pas totalement dans cette étude. Les travaux de ces auteurs ont porté sur une période plus longue (au moins $10 \mathrm{j}$ ) ; de plus, les températures basses ont parfois été appliquées à l'ensemble du végétal (BLONDON et al., 1980). Les résultats présentés ici permettent néanmoins d'avancer que l'«effet ajustement» de températures basses appliquées au niveau racinaire est plus fort pour le type denté $(\mathrm{WH} \times \mathrm{WJ})$ que pour le type corné $(\mathrm{F} 7 \times \mathrm{F} 2)$.

TABLEAU 4

Résultats d'analyses de variance à 2 critères portant sur quelques paramètres de croissance (** : l'effet est significatif au niveau $1 \%$; *: l'effet est significatif au niveau $5 \%$ ).

Two-way variance analysis of growth parameters $\left(^{* *}:\right.$ significant effect at $1 \%$ level ${ }^{*}$ : significant effect at $5 \%$ level).

\begin{tabular}{cccc} 
& & \multicolumn{3}{c}{ Type d'effet } \\
\hline Paramètre & Température & Génotype & Interaction \\
\hline SF & NS & $*$ & $* *$ \\
\hline PST & $* *$ & $* *$ & $* *$ \\
\hline PSR & $* *$ & $* *$ & NS \\
\hline PSPA & $* *$ & NS & $* *$ \\
\hline PSR/PSPA & $* *$ & $* *$ & $* *$ \\
\hline PSR/PST & $* *$ & $* *$ & $* *$ \\
\hline PST/SF & $* *$ & $* *$ & $* *$ \\
\hline TET & $* *$ & NS & $* *$ \\
\hline TER & $* *$ & $* *$ & NS \\
\hline TEPA & $* *$ & $* *$ & $*$ \\
\hline \hline
\end{tabular}

\section{CONCLUSION}

Il apparaît donc que la moins bonne tolérance aux basses températures constatée au champ de $\mathrm{WH} \times \mathrm{WJ}$ par rapport à $\mathrm{F} 7 \times \mathrm{F} 2$ soit en relation, en ce qui concerne les racines, avec :

- une limitation plus grande du métabolisme énergétique général ;

- une forte modification de l'état hydrique des tissus.

D'après les résultats de la littérature que nous avons rapportés aux chapitres I et III, on peut penser qu'il s'agit d'une règle générale pour la tolérance au froid du maïs. 
Cependant, pour le confirmer et aboutir à des tests relativement simples sur la sensibilité au froid, il faudrait étendre l'étude présentée à un éventail variétal plus large, éventail proposé par les généticiens d'après leur expérience au champ.

Nous avons souligné la limitation du métabolisme énergétique primaire par la température. Dans les conditions naturelles, quand se révèle la tolérance ou la non-tolérance au froid lors de l'installation de la culture, le sol est souvent saturé en eau. Cette saturation entraîne, pour le moins, une hypoxie au niveau des racines dont la respiration est alors limitée par le manque d'oxygène. Ainsi, les observations agronomiques sur la tolérance au froid dans les régions septentrionales proviennent probablement d'une limitation du métabolisme énergétique général liée conjointement à des températures peu élevées et à une hypoxie.

Les résultats obtenus montrent que les différences de comportement s'établissent très rapidement. Les méthodes présentées dans cette étude pourraient donc servir de base à un criblage variétal pour la sensibilité au froid, au cours des premiers stades de végétation.

Reçu le 15 novembre 1984. Accepté le 1er avril 1985.

\section{RÉFÉRENCES BIBLIOGRAPHIQUES}

Adam Mc J. H., Hayes P., 1981. The effect of shoot-zone temperature and root-zone temperature on the early growth and development of Zea mays cv. 'Maris Carmine'. Record Agric. Res., 29, 39-45.

Akimova G. P., Rodchenko O. P., 1977. Change in peroxidase activity in corn root cells at low positive temperature. Dokl. bot. Sci., 234, 75-77.

Allmaras R. R., Burrows W. C., Larson W. E., 1964. Early growth of corn as affected by soil temperature. Soil Sci. Am. Proc., 28, 271-275.

Beauchamp E., Lathwell J., 1966, Root-zone temperature effects on the vascular development of adventious roots in Zea mays. Bot. Gaz., 127, 153-158.

Blondon F., Clabault G., Rainguez M., 1980. Action d'une température fraîche $\left(10^{\circ} \mathrm{C}\right)$ appliquée au stade jeune sur la croissance et l'activité de deux variétés précoces de maïs. Ann. Amélior. Plantes, $30,399-410$.

Blondon F., Clabault G., Rainguez M., 1981. Déplacement de l'optimum thermique de l'activité photosynthétique, sous l'action d'un traitement à température fraîche $\left(10^{\circ} \mathrm{C}\right)$, chez le maïs : différenciation de deux variétés. C. R. Acad. Sci. Paris, Série III, 292, 149-152.

Bourdu R., Prioul J. L., 1974. Réponses photosynthétiques de type adaptatif aux climats lumineux de croissance: étude théorique, applications et diagnostic précoce. Physiol. Vég., 12, 35-51.

Bravo F. P., Uribe E. G., 1981. Temperature dependence of the concentration kinetics of absorption of phosphate and potassium in corn roots. Plant Physiol., 67, 815-819.

Burbanova R. S., Rodchenko O. P., 1980. Effect of lowered temperature on protein synthesis in primary maize roots (en russe). Sibirskii Institut Fiziologii i Biokhimii Rastenii, Irkutsk, USSR, 25-33 (Abstr. in Field Crop Abs., 1982, 8781).

Cauderon A., 1958. Les maïs hybrides en France. Etude de la précocité. Ann. Amélior. Plantes, 8, 273-289.

Carey W. R., Berry J. A., 1978. Effects of low temperature on respiration and uptake of rubidium ions by excised barley and corn root. Plant Physiol., 61, 858-860.

Chaillou S., Blondon F., Schiedecker D., 1976. Contribution à l'étude de l'action d'une température basse sur la croissance et l'ali- mentation minérale d'un hybride précoce de maïs. C. $R$. Acad. Agric. Fr., 62, 714-724.

Chatenoud J., 1982. Recherches sur la sélection en vue d'améliorer la résistance à la sécheresse du tournesol (Helianthus annuus) par croisement avec Helianthus argophyllus. Mémoire de fin d'études de l'I.S.A.B. (Beauvais), $44 \mathrm{p}$.

Crawford R. M. M., Huxter T. J., 1977. Root growth and carbohydrate metabolism at low temperature. J. exp. Bot., 28, 917-925.

Derieux M., Bonhomme R., 1982. Heat unit requirements for maize hybrids in Europe. Results of the European FAO sub-network. I. Sowing-silking period. Maydica, XXVII, 59, 77.

Duburcq J. B., Bonhomme R., Derieux M., 1983. Durée des phases végétative et reproductrice chez le maïs. Influence du génotype et du milieu. Agronomie, 3, 941-946.

Frossard J. S., 1976. Relations entre l'éclairement des feuilles et l'absorption d'oxygène par les racines chez le tournesol (Helianthus annuus L.). Ann. Agron., 27, 435-445.

Frossard J. S., 1981. Comparaison de la respiration racinaire chez des espèces à métabolisme phytosynthétique de type $\mathrm{C} 3$ et chez des C4 au cours d'un nycthémère. C. R. Acad. Sci., Paris, 292, série D, 1033-1035.

Fukai S., Silsbury J. H., 1977. Responses of subterranean clover communities to temperature. II Effects of temperature on dark respiration rate. Aust. J. Plant Physiol., 4, 159-167.

Grobbelaar W. P., 1963. Response of young maize plants to root temperatures. Meded. Landbouwhogeschool, Wageningen, 63, 1-71.

Kleinendorst A., Brouwer R., 1970. The effect of temperature of the root medium and of the growing point of the shoot on growth, water content and sugar content of maize leaves. Neth. J. agric. Sci., 18, 140-148.

Stamp P., 1978. Spross und Wurzelmerkmale junger Maispflanzen Verschiedener Kornform in Abhängigkeit von der Temperatur. (Shoot and root characteristics of young maize plants of various grain types in relation to temperature). Z. Acker-und Pflanzenbau, $148,99-108$.

Sutcliffe J., 1977. Plants and temperature. Sussex Univ., Brighton, $57 \mathrm{p}$. 